8. Kilic A, Greene S, Rojas L, et al. Detectoin of $m c r-1$ gene in a clinical Escherichia coli strain in North Carolina: first report. J Glob Antimicrob Resist 2021:1554-1156.

9. Mavrici D, Yambao J, Lee B, Quinones B, He X. Screening for the presence of $m c r-1 / m c r-2$ genes in Shiga toxin-producing Escherichia coli recovered from a major produce-producing region in California. PLoS One 2017;12: e0187827.

10. Meinersmann R, Ladely S, Plumblee J, Cook K, Thacker E. Prevalence of $m c r-1$ in the cecal contents of food animals in the United States. Antimicrob Agents Chemother 2017;61:e02244-16.

\title{
Is it time for us to account for the impact of coronavirus disease 2019 (COVID-19) on healthcare-associated infections?
}

\author{
Mohamad G. Fakih MD, MPH ${ }^{1}$ (1), Lisa K. Sturm MPH, $\mathrm{CIC}^{1}$, Rama V. Thyagarajan MD, MPH, $\mathrm{ClC}^{2}$ (1) and \\ Kristin E. Mondy MD, FACP ${ }^{2}$ (1) \\ ${ }^{1}$ Quality Department, Clinical and Network Services, Ascension, St. Louis, Missouri and ${ }^{2}$ Division of Infectious Diseases, Department of Medicine, \\ Dell Medical School, University of Texas at Austin, Austin, Texas
}

To the Editor-We read with interest the recent study by Weiner-Lastinger et $\mathrm{al}^{1}$ on the impact of coronavirus disease 2019 (COVID-19) pandemic on nationally reportable healthcare-associated infections (HAIs). This is the largest and most comprehensive study evaluating national performance on HAIs during the pandemic using National Healthcare Safety Network (NHSN) data. They compared the standardized infection ratios (SIRs) for reportable infections in different quarters of 2019 to their corresponding quarters in 2020. Overall, the COVID-19 pandemic was associated with significant increases in many reportable HAIs. The changes varied per quarter, but by the fourth quarter of 2020 , there was a $47 \%$ increase in central line-associated bloodstream infections (CLABSI), a $34 \%$ increase in LabID hospital-onset (HO) methicillin-resistant Staphylococcus aureus (MRSA) bacteremia, a 45\% increase in ventilator associated events, and a 19\% increase in catheter-associated urinary tract infections (CAUTI). On the other hand, there was a decrease in colon surgery SSIs (8\%) and abdominal hysterectomy SSIs (13\%), as well as LabID HO Clostridioides difficile infection (CDI, 5.5\%). The effect of the COVID-19 pandemic during the second and third quarters of 2020 was less visible. The pandemic was not as widespread compared to the last quarter of the previous year. These findings are important because they reflect the sensitivity of the different NHSN measures to the pandemic.

The pandemic has revealed future opportunities when evaluating and following the progress of HAIs in US hospitals. First, our current measures and their risk adjustment may not adequately reflect the impact of the COVID-19 pandemic on patient risk (whether intrinsic or extrinsic factors). ${ }^{2}$ Traditionally, the risk adjustment incorporated into the SIR includes facility, unit characteristics, and a few patient factors. ${ }^{3}$ The current baseline has been adjusted to the performance in 2015 and assumes that no large changes in population characteristics have occurred. Second, incorporating community and preferably hospital and unit level COVID-19 prevalence as a factor may help better evaluate HAI risk adjustment in corresponding hospitals. COVID-19 surges and inpatient caseloads have been associated with higher mortality ${ }^{4}$ and CLABSI. ${ }^{5}$ Reviewing the national study, ${ }^{1}$ CLABSI

Author for correspondence: Mohamad G. Fakih, E-mail: Mohamad.Fakih@ascension.org Cite this article: Fakih MG, et al. (2022). Is it time for us to account for the impact of coronavirus disease 2019 (COVID-19) on healthcare-associated infections?. Infection Control \& Hospital Epidemiology, 43: 1960-1961, https://doi.org/10.1017/ice.2021.450 and HO-MRSA bacteremia for specific states like Michigan and New Jersey exhibited significant increases in the second quarter of 2020 but not the third quarter, consistent with the early surges. Moreover, the currently submitted measures do not identify the proportion of patients with COVID-19 developing HAI infection (reporting COVID-19 status with each HAI remains optional). Third, the SIR does not account for drastic changes in device utilization or changes in volumes of procedures. A few years ago, the CDC introduced the standardized utilization ratio (SUR) to evaluate device utilization. ${ }^{6}$ Compared with 2019 , central-line and urinary catheter utilization increased by $7 \%$ and $9 \%$, respectively, whereas a much more pronounced change in ventilator SUR, up to $30 \%$, was observed. These findings underscore the importance of devising new measures that account for the overall population risk. $^{7}$ Finally, the analysis of the national data was limited by including only hospitals with complete surveillance data. Over the first 2 quarters of 2020, there was a $12 \%-14 \%$ reduction in reporting CLABSIs, CAUTIs, HO-CDIs, and HO-MRSA bacteremia cases. On the other hand, hospitals had larger reductions in reporting SSIs (colon 25\%-27\% and abdominal hysterectomy $32 \%-36 \%)$. The interruption in reporting was more notable at the state level, with $61 \%$ of hospitals in the state of New Jersey and $41 \%$ in the state of New York not reporting CLABSI for the second quarter of 2020. The attrition in reporting may underestimate the impact of the pandemic on HAIs because hospitals with large numbers of COVID-19 patients (and subsequently higher HAI rates) may have ceased reporting due to other priorities.

Examining specific HAIs, the pandemic has helped us better understand the susceptibility of the measures to significant changes in patient characteristics and care. For example, CLABSI is a very sensitive measure to changes in population and practices. Changes in central-line care, duration of use, and patient characteristics heavily affect its outcomes. We have reported that hospitals with COVID-19 patients representing $>10 \%$ of admissions had 2.4 times more CLABSI events than those with $<5 \%$ COVID-19 cases. Proportionately, COVID-19 patients had $>5$ times more CLABSI events than patients not infected with COVID-19. Similarly, HO-MRSA bacteremia events may be heavily affected by the increase in intravascular device infections, ventilator-associated pneumonias, and hospital-acquired pneumonias in COVID-19-infected patients. ${ }^{8}$ On the other hand, the NHSN CAUTI definition is more dependent on culturing practices, preexisting prevalence of bacteriuria, antimicrobial pressure, and is less susceptible 
to device maintenance. ${ }^{5}$ Colon and abdominal hysterectomy SSIs did not show an increase contrary to the expectation with higher risk, less elective, and more emergent surgeries being performed. There was a large decrease in hospitals reporting SSIs, a sign of the complexity associated with surgical surveillance in times of limited resources.

In conclusion, the COVID-19 pandemic has helped us better understand the strengths and limitations of our national surveillance measures for HAIs. We also need to apply a more rigorous risk adjustment for patient conditions, to have the flexibility to adjust for the changing landscape that a pandemic brings, to easily incorporate emerging factors that affect processes of care, and to devise new surveillance measures that are easily captured electronically. More importantly, it is time to reexamine whether our current measures provide us optimal evaluations of the progress toward preventing infections and the mitigation steps necessary to reduce them.

\section{Acknowledgments.}

Financial support. No financial support was provided relevant to this article. Conflicts of interest. All authors report no conflicts of interest relevant to this article.

\section{References}

1. Weiner-Lastinger LM, Pattabiraman V, Konnor RY, et al. The impact of coronavirus disease 2019 (COVID-19) on healthcare-associated infections in 2020: a summary of data reported to the National Healthcare Safety Network. Infect Control Hosp Epidemiol 2021. doi: 10.1017/ ice.2021.362.

2. Salmasian H, Beloff J, Resnick A, et al. Rethinking standardised infection rates and risk adjustment in the COVID-19 era. BMJ Qual Saf 2021; 30:588.

3. The NHSN standardized infection ratio (SIR). A guide to the SIR. Centers for Disease Control \& Prevention website. https://www.cdc.gov/ nhsn/pdfs/ps-analysis-resources/nhsn-sir-guide.pdf. Accessed September 23, 2021.

4. Kadri SS, Sun J, Lawandi A, et al. Association between caseload surge and COVID-19 survival in 558 US hospitals, March to August 2020. Ann Intern Med 2021;174:1240-1251.

5. Fakih MG, Bufalino A, Sturm L, et al. Coronavirus disease 2019 (COVID-19) pandemic, central-line-associated bloodstream infection (CLABSI), and catheter-associated urinary tract infection (CAUTI): the urgent need to refocus on hardwiring prevention efforts. Infect Control Hosp Epidemiol 2021. doi: $10.1017 /$ ice.2021.70.

6. The NHSN standardized utilization ratio (SUR). A guide to the SUR. Centers for Disease Control and Prevention website. https://www.cdc.gov/nhsn/pdfs/psanalysis-resources/nhsn-sur-guide-508.pdf. Accessed September 23, 2021.

7. Fakih MG, Huang R-H, Bufalino A, et al. The case for a population standardized infection ratio (SIR): a metric that marries the device SIR to the standardized utilization ratio (SUR). Infect Control Hosp Epidemiol 2019;40:979-982.

8. Grasselli G, Scaravilli V, Mangioni D, et al. Hospital-acquired infections in critically ill patients with COVID-19. Chest 2021;160:454-465.

\title{
Outbreak of central-line-associated bloodstream infections (CLABSIs) amid the coronavirus disease 2019 (COVID-19) pandemic associated with changes in central-line dressing care accompanying changes in nursing education, nursing documentation, and dressing supply kits
}

\author{
Shiv A. Patel BS ${ }^{1}$ (1), Ajay K. Rajan BA ${ }^{1}$ (1), Ahad Azeem MBBS ${ }^{2,3}$, Irene L. Newquist RN, MSN², Lesley L. Royal RN, MSN², \\ Kimberly S. Hemrick MT(ASCP), MHA ${ }^{2}$, Gia Thinh D. Truong BS ${ }^{1}$ (1) , Zachary A. Creech BS ${ }^{1}$, Faran Ahmad MBBS ${ }^{2,3}$ (D) and \\ Marvin J. Bittner MD ${ }^{2,3}$ (1) \\ ${ }^{1}$ Creighton University School of Medicine, Omaha, Nebraska, ${ }^{2}$ Veterans Health Administration Nebraska-Western lowa Health Care System, Omaha, Nebraska and \\ ${ }^{3}$ Department of Internal Medicine, Creighton University School of Medicine, Omaha, Nebraska
}

To the Editor-Central-line-associated bloodstream infections (CLABSIs) result in roughly 28,000 preventable deaths each year at an average cost of $\$ 45,814$ per infection. ${ }^{1}$ Central-line bundles

Author for correspondence: Marvin J. Bittner MD, E-mail: marvin.bittner@va.gov PREVIOUS PRESENTATION. This submission includes material previously presented as poster 774 at ID Week 2021 on September 29, 2021, held virtually. The associated abstract was published as follows: Azeem A, Newquist IL, Royal LL, Hemrick KS, Creech ZA, Patel SA, Rajan AK, Truong GD, Ahmad F, Bittner MJ. Outbreak of central-line-associated bloodstream infections (CLABSIs) amid the COVID-19 pandemic associated with changes in central-line dressing care accompanying changes in nursing education, nursing documentation, and dressing supply kits. Open Forum Infect Dis 2021;8 suppl 1: abstract S484.

Cite this article: Patel SA, et al. (2022). Outbreak of central-line-associated bloodstream infections (CLABSIs) amid the coronavirus disease 2019 (COVID-19) pandemic associated with changes in central-line dressing care accompanying changes in nursing education, nursing documentation, and dressing supply kits. Infection Control \& Hospital Epidemiology, 43: 1961-1963, https://doi.org/10.1017/ice.2022.89 have been shown to achieve zero CLABSIs. ${ }^{2}$ The Centers for Disease Control and Prevention (CDC) guidelines recommend dressings impregnated with chlorhexidine gluconate $(\mathrm{CHG})$ for patients aged $\geq 18$ years to protect the site of insertion due to their effectiveness in preventing CLABSI. ${ }^{3,4}$

Our hospital reported no cases of CLABSI for 18 months between April 2019 and November 2020. However, from November 2020 to March 2021, we encountered at least 1 CLABSI each month for a total of 7 CLABSIs in this 5-month period. This report describes our investigation into the outbreak and its principal findings, which were largely related to changes in the use of CHG-impregnated dressings.

\section{Methods}

Infection preventionists at the Veterans Health Administration Nebraska-Western Iowa Health Care System Omaha Veterans 